\title{
The Sensitive Period for Auditory Localization in Barn Owls Is Limited by Age, Not by Experience
}

\author{
Eric I. Knudsen and Phyllis F. Knudsen \\ Department of Neurobiology, Stanford University School of Medicine, Stanford, California 94305
}

Early in life, the barn owl passes through a sensitive period during which it can interpret and make use of abnormal auditory cues for accurate sound localization. This capacity is lost at about 8 weeks of age, just after the head and ears reach adult size (Knudsen et al. 1984a). The end of the sensitive period could be triggered either by an age-dependent process or by the exposure of the auditory system to stable or adult-like cues. To distinguish between these alternatives, we subjected baby owls to constant abnormal cues (chronic monaural occlusion) or to frequently changing abnormal cues (alternating monaural occlusion) throughout the sensitive period.

In the first group of animals $(n=2)$, one ear was plugged continuously until 73 or $79 \mathrm{~d}$ of age, respectively, and then the earplug was switched to the opposite ear. Although these animals adjusted sound localization accuracy during the initial chronic monaural occlusion, they could not localize sounds at all after the earplug was switched to the opposite ear, and they remained unable to localize sounds as long as the opposite ear remained occluded ( 7 and 27 weeks, respectively). When the second monaural occlusion was finally removed, both birds localized sounds with errors that were similar to the errors they exhibited immediately after removal of the first monaural occlusion. One bird that was 127-d-old at the time the second earplug was removed corrected its localization error; the other bird, 250-d-old when the second earplug was removed, did not. These results demonstrate that abnormal associations between auditory cues and sound-source locations can remain stable for long periods of time without functional validation, and that the critical period for adjusting auditory localization accuracy can end by a certain age even though an animal cannot localize sounds. However, because these animals could not localize sounds once the earplug was switched to the opposite ear, these data did not answer the question of whether or not the sensitive period had been extended.

Therefore, in a second group of baby owls $(n=4)$, we switched the earplug from one ear to the other every second day. This high rate of alternating monaural occlusion ensured that the animals never adjusted completely to the abnormal cues. Alternating occlusion continued until the birds were older than $60 \mathrm{~d}$ of age and was followed by chronic monaural occlusion to test the ability of the animals to adjust auditory localization. None of these birds adjusted auditory localization when exposed to chronic monaural occlusion. Thus, despite exposure to abnormal

Received Aug. 12, 1985; revised Nov. 12, 1985; accepted Jan. 6, 1986.

We thank Drs. Sascha du Lac, Steven Esterly, Masakazu Konishi, Stephen Lisberger, John Middlebrooks, and Carla Shatz for reviewing the manuscript, and Brenda Robertson for typing the manuscript. This work was supported by grants from the March of Dimes (1-863), Sloan Foundation, the National Institutes of Health (R01 NS 16099-06), and a Neuroscience Development Award from the McKnight Foundation.

Correspondence should be addressed to Eric I. Knudsen, Ph.D., Department of Neurobiology, Stanford University School of Medicine, Sherman Fairchild Science Building, D200, Stanford, CA 94305.

Copyright (C) 1986 Society for Neuroscience $0270-6474 / 86 / 071918-07 \$ 02.00 / 0$ and changing auditory cues, the sensitive period still ended at about 8 weeks of age. On the basis of these data, we conclude that the end of the sensitive period is triggered by a process that is essentially, if not exclusively, age-dependent.

To localize sounds, an animal must associate sets of monaural and binaural cues with appropriate locations in space. In barn owls, scnsory expcricnce shapes thesc associations during restricted periods in development (Knudsen, 1985; Knudsen et al., 1982, 1984a, b). During the sensitive period, which ends at about 8 weeks of age, owls can alter their interpretations of auditory cues and learn to use abnormal cues to localize sounds accurately (Fig. 1A); older owls lose this capacity. If owls that have learned to use abnormal cues are prevented from experiencing normal sound-localization cues throughout the critical period, which ends at about 29 weeks of age, they never acquire accurate localization after normal auditory cues are restored (Fig. 1B).

The end of the sensitive period is well defined. In a previous study, an owl that was monaurally occluded at $55 \mathrm{~d}$ of age adjusted to the abnormal auditory cues imposed by the earplug, whereas another bird that was monaurally occluded at $60 \mathrm{~d}$ of age did not (Knudsen et al., 1984a). In the same study, we noted that the head and external ears reached adult size just 2 weeks before the sensitive period closed. Since the sizes and shapes of these structures are directly responsible for sound localization cues, owls do not experience stable, adult cues until their growth stops. This coincidence suggests the hypothesis that exposure of the auditory system to stable and/or adult localization cues causes the sensitive period to end. The alternative hypothesis is that the sensitive period is terminated by an age-dependent process.

We have tested these hypotheses by exposing young barn owls to abnormal and changing cues throughout the first few months of life. If experience with either adult-like or stable cues is required to end the sensitive period, then the sensitive period should have remained open in these animals. It did not, supporting the hypothesis that the sensitive period for sound localization is ended by an age-dependent process.

\section{Materials and Methods}

Six barn owls (Tyto alba) were subjected to various regimens of alternating monaural occlusion (Table 1). Ear occlusion was accomplished by suturing a foam rubber plug (E.A.R.; Cabot Corporation) into the ear canal while the owl was anesthetized with halothane and nitrous oxide. Two owls (Nos. 1 and 2) underwent a single reversal of the earplug: Each had one ear plugged for $50 \mathrm{~d}$, then the opposite ear plugged for $49 \mathrm{~d}$ (owl No. 1) or $178 \mathrm{~d}$ (owl No. 2). A group of 4 owls (Nos. 36) underwent earplug reversals every other day for periods of 47-85 d, followed by chronic monaural occlusion lasting for 103-127 d. In these animals, the temporary earplugs were installed without anesthetics by tying them to sutures that were attached permanently around the entrances to the ear canals.

The experimental conditions and methods were identical to those 
A

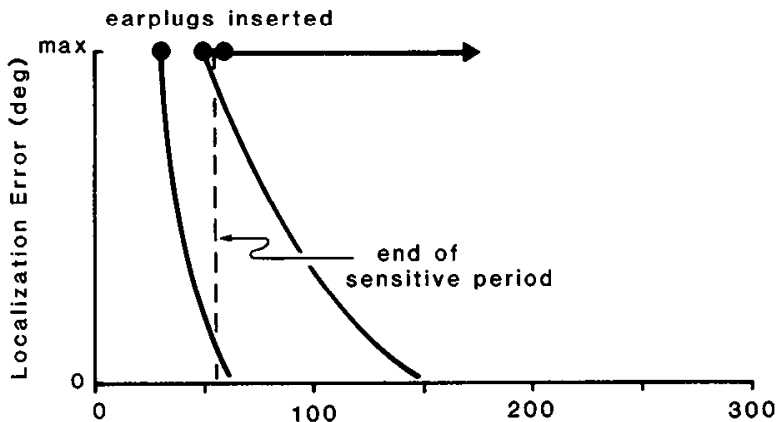

B

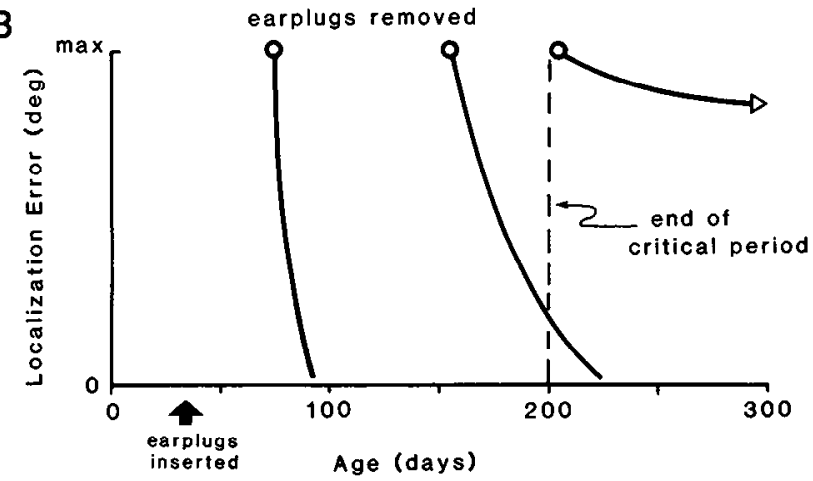

Figure 1. Sensitive and critical periods in the development of sound localization in the barn owl, as defined by data from previous monaural occlusion experiments. Sound-localization error is plotted against age for 3 representative, chronically occluded animals following carplug insertion $(A)$ and earplug removal $(B)$. The data in $A$ are from owls No. 4,16 , and 17 in Knudsen et al. (1984a); the data in $B$ are from owls No. 1, 5, and 7 in Knudsen et al. (1984b). Owls adjust to abnormal auditory cues and correct their sound localization errors only when monaural occlusions are imposed within the sensitive period. In $B$, owls raised with one ear occluded acquire accurate sound localization following removal of the earplug only if the earplug is removed before the end of the critical period (dashed lines).

described previously (Knudsen et al., 1984a). Sound localization was assessed from head-orientation responses to auditory and to visual stimuli. The stimuli were presented in a darkened sound-attenuating chamber with the owl perched in the center. Auditory stimuli were generated by a $4 \mathrm{~cm}$ speaker, and visual stimuli by a light-emitting diode that was centered in the cone of the speaker. The speaker and diode were attached to a carriage that moved by remote control along a semicircular track. The track itself rotated about a vertical axis so that the speaker and diode could be positioned at virtually any location with respect to the owl.

The behavioral paradigm required that the owl orient its head to auditory or visual stimuli in order to receive a food reward. The reward was not contingent upon the accuracy of the response, only upon its latency and speed. To quantify the orientation responses, a small mirror was attached to a clip cemented to the skull, and a collimated infrared light was directed at the animal. The mirror reflected an IR beam onto a calibrated screen $\left(0.5^{\circ}\right.$ increments) that covered the front wall. The position of the beam on the screen was monitored with a video camera and was viewed on a $30 \times 38 \mathrm{~cm}$ television screen.

The auditory stimulus consisted of repetitive noise bursts of variable amplitude, duration, and repetition rate. The visual stimulus was a sustained glow from the diode. In both cases, the stimulus was presented for up to 2 sec, which was long enough to allow the owl to maximize its accuracy. If the animal did not respond, the stimulus was turned off and a waiting period of about $30 \mathrm{sec}$ ensued. Typically, however, the animal responded immediately with a quick head movement and a sustained fixation. The coordinates of the orientation response were then recorded, a reward light was activated, and a food reward delivered. The reward criteria were that the owl turn its head and fixate on a point during the stimulus. Because the reward was not contingent upon the
Table 1. Auditory histories of the owls

\begin{tabular}{|c|c|c|c|c|}
\hline \multirow[b]{2}{*}{ Owl } & \multicolumn{2}{|c|}{ Initial earplug } & \multicolumn{2}{|c|}{ Final earplug } \\
\hline & Ear & Age (d) & Ear & Age (d) \\
\hline 1 & $\mathbf{R}$ & $30-79$ & $\mathbf{L}$ & $79-127$ \\
\hline 2 & $\mathbf{L}$ & $24-73$ & $\mathbf{R}$ & $73-250$ \\
\hline 3 & $\mathbf{R} \rightleftharpoons \mathbf{L}^{a}$ & $16-62$ & $\mathbf{R}$ & $62-168$ \\
\hline 4 & $\mathbf{R} \rightleftharpoons \mathbf{L}^{a}$ & $16-70$ & $\mathbf{R}$ & $70-172$ \\
\hline 5 & $\mathrm{R} \rightleftharpoons \mathrm{L}^{a}$ & $16-84$ & $\mathbf{R}$ & $84-210$ \\
\hline 6 & $\mathbf{R} \rightleftharpoons \mathbf{L}^{a}$ & $16-100$ & $\mathbf{R}$ & $100-218$ \\
\hline
\end{tabular}

a A monaural plug was moved from one ear to the other every other day.

accuracy of the response, it did not bias the responses. After every trial the speaker and diode were moved to a new, random location.

A test session included 15-25 responses to the auditory stimulus and an equal number of responses to the visual stimulus. For each trial, the location of the stimulus and the coordinates of the final head orientation were recorded. The orientation responses were expressed in degrees of azimuth (left-right) and elevation (up-down) relative to the location of the stimulus. From these data, the mean and SD of the responses both to the auditory and visual stimuli were calculated.

Because normal owls orient almost identically to auditory and visual stimuli, sound localization accuracy (the closeness of the response to
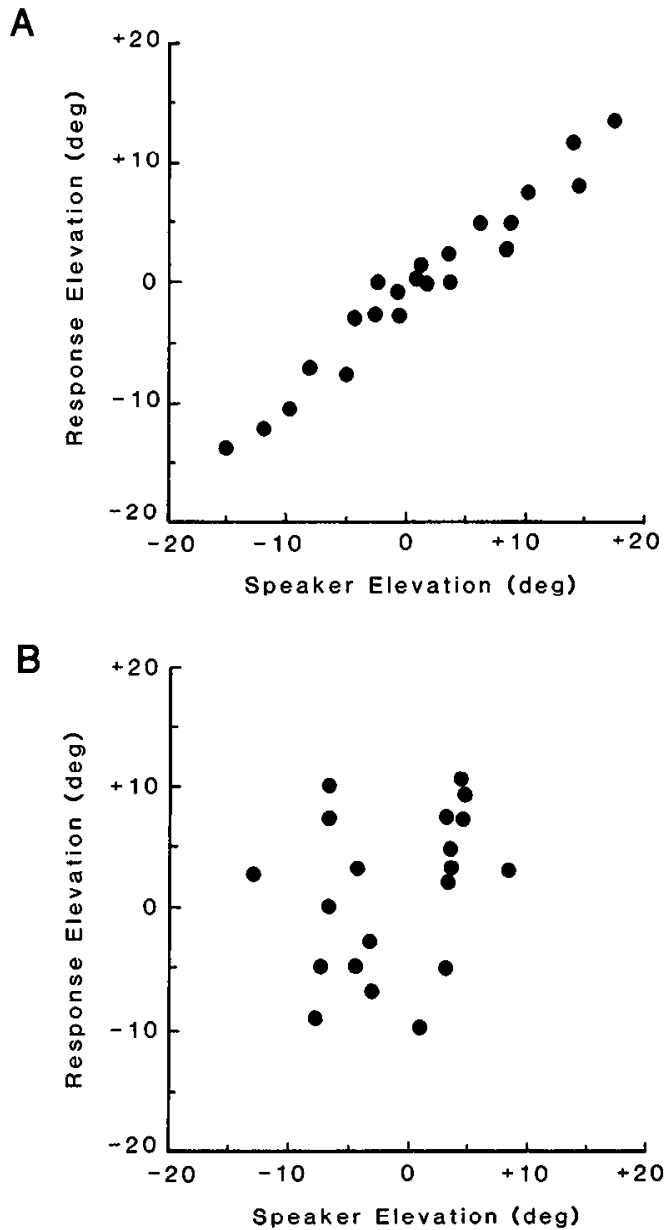

Figure 2. Sound-source position versus head orientation for days on which owl No. 1 could $(A)$ and could not $(B)$ localize sounds based on the criteria described in Materials and Methods. The data in $A$ were collected on day 154; the slope is 0.7 and $F_{(21.21)}=140$ (significant at the $p<0.01$ level). The data in $B$ were collected on day 113; the slope is 0.5 and $F_{(19,19)}=2.2$ (not significant). 
Figure 3. The effect of a single reversal of monaural occlusion on sound localization in owl No. 1 . In $A$, mean localization error, which is the magnitude of the average localization error (both the azimuthal and elevational components), is plotted against age. The auditory history of the bird is indicated along the abscissa. Xs, Localization errors measured with one ear plugged; open circles, measurements made with both ears open. The inability of the bird to localize sounds during the period of left ear occlusion was based on the criteria described in Materials and Methods. In $B$, raw data from particular days are shown. Filled circles, Individual orientation responses to acoustic stimuli plotted in degrees of azimuth and elevation relative to the location of the speaker, which is indicated by the circled cross. Notice that the performance of the owl immediately before (day 79 ) and after (day 127) left ear occlusion is virtually the same.
A

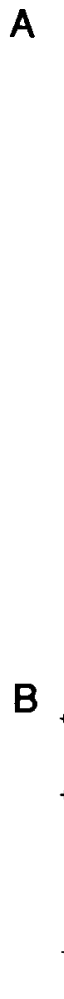

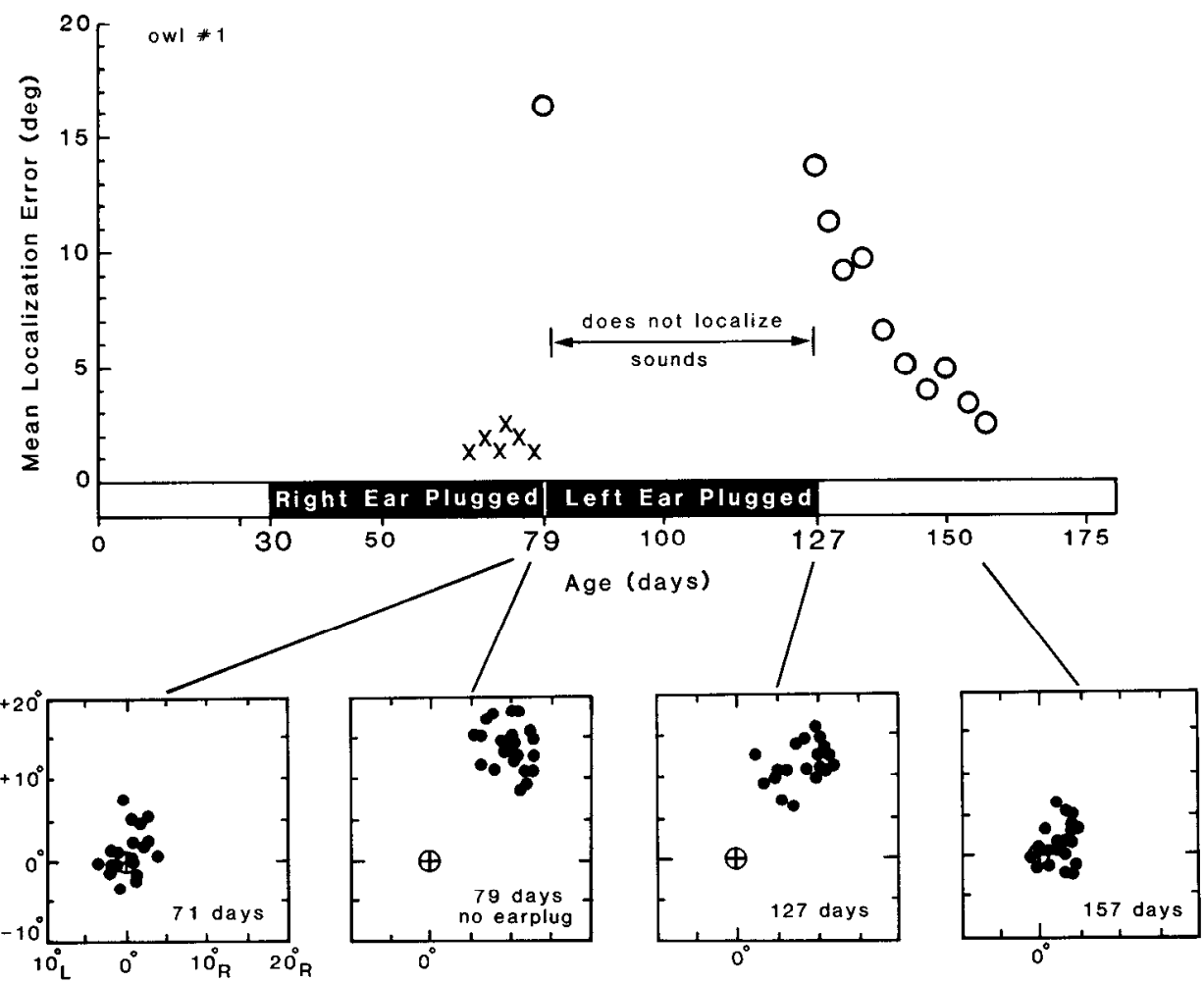

the true location of the sound source) was mcasurcd as the difference between the mean auditory and mean visual responses. Using this approach, we found that the average magnitude of the sound localization error of 6 normal owls studied previously was $1.4 \pm 0.8^{\circ}$ (SEM) in azimuth and $1.2 \pm 0.9^{\circ}$ in elevation (Knudsen et al., 1984a). We chose as our criterion for "normal" accuracy mean values that were within 2 SEM of these control values. Thus, average localization errors that were less than $3.0^{\circ}$ in each dimension were considered normal. "Mean localization error" is the magnitude of the mean error, which combines the azimuthal and elevational components.

Sound localization precision (the reliability of the response) was quan- tified by the SDs of the responses to the auditory stimulus. The average precision among 6 normal owls was $1.8 \pm 0.6^{\circ}$ in azimuth and $2.0 \pm$ $0.7^{\circ}$ in elevation (Knudsen et al., 1984a). Our criterion for "normal" precision was based on an $F$ test $(p<0.01)$ of whether the experimental variance was larger than the control variance. For most samples, localization precision was normal when the SD was $3.0^{\circ}$ or less in azimuth and $3.7^{\circ}$ or less in elevation.

Under certain circumstances, it was apparent that an animal was unable to localize the auditory stimulus in one or both dimensions. Although the SDs of the responses were always large in such cases, a large SD alone did not mean that the animal was not localizing the
Figure 4. The effect of a single reversal of monaural occlusion on sound $B$ localization in owl No. 2. The format and symbols are the same as those used in Figure 3. Notice that the performance of the owl immediately before (day 73) and after (day 250) right ear occlusion is virtually the same, and that this owl never recovered accurate sound localization after removal of the right earplug.

A
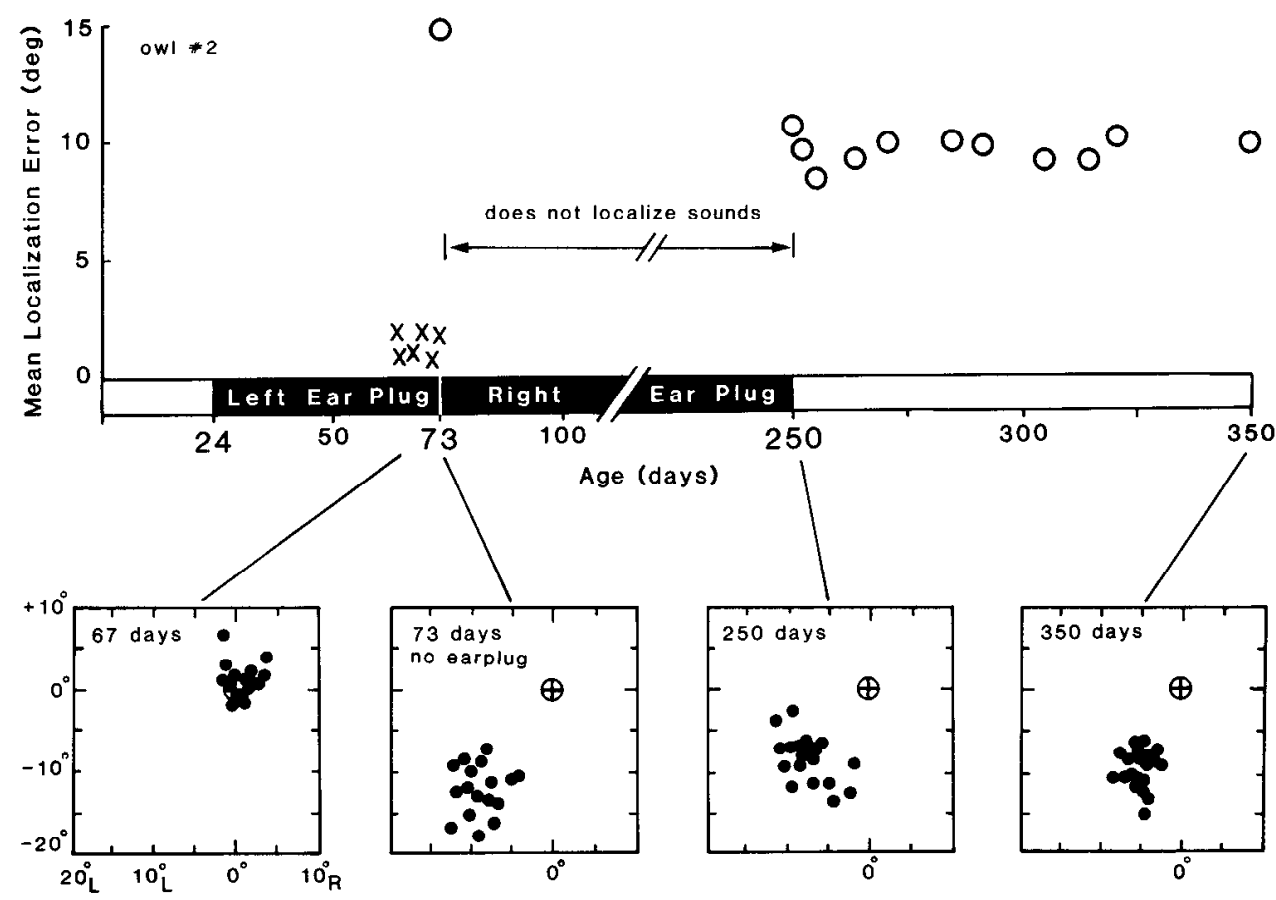


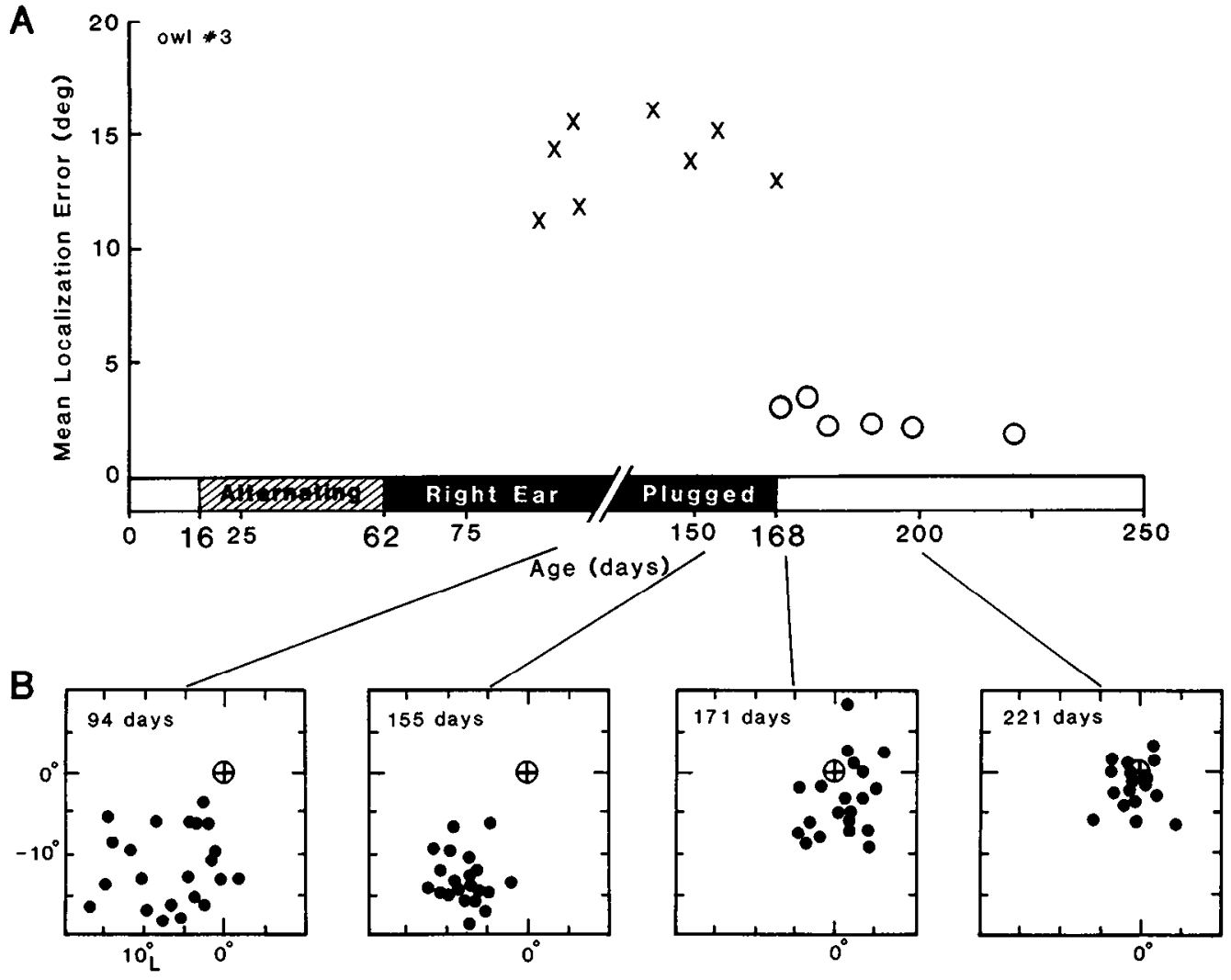

Figure 5. The effect of alternating monaural occlusion followed by chronic monaural occlusion on sound localization in owl No. 3. In $A$, mean localization error is plotted against age. The auditory history of the bird is indicated along the abscissa. The period of alternating monaural occlusion, during which the earplug was switched every other day, is represented by $\mathrm{di}$ agonal hatching. Xs indicate localization errors measured with one ear plugged; open circles indicate measurements with both ears open. In $B$, raw data from particular days are shown. Note that after the right earplug was removed, the owl's error was small, although its precision was poor. sound source. To determine whether the animal was localizing, we performed a linear regression of the location of the speaker versus the location of the response for each spatial dimension (Fig. 2). For owls that were localizing well, points clustered tightly about a line with a slope of 1.0. For owls that were localizing poorly, the slope decreased and the fit to a line deteriorated. When a slope was not significantly different from zero ( $F$ test; $p<0.01$ ), we considered the owl not to be localizing sounds in that dimension and no accuracy or precision values were computed.

\section{Results}

Single earplug reversals

In this experiment, we investigated whether animals that have adjusted their sound localization using one set of abnormal cues can adjust again to an entirely different set of abnormal cues. Owls No. 1 and 2 were monaurally occluded initially at 30 and $24 \mathrm{~d}$ of age, respectively (Table 1; owl No. 1, right earplug; owl

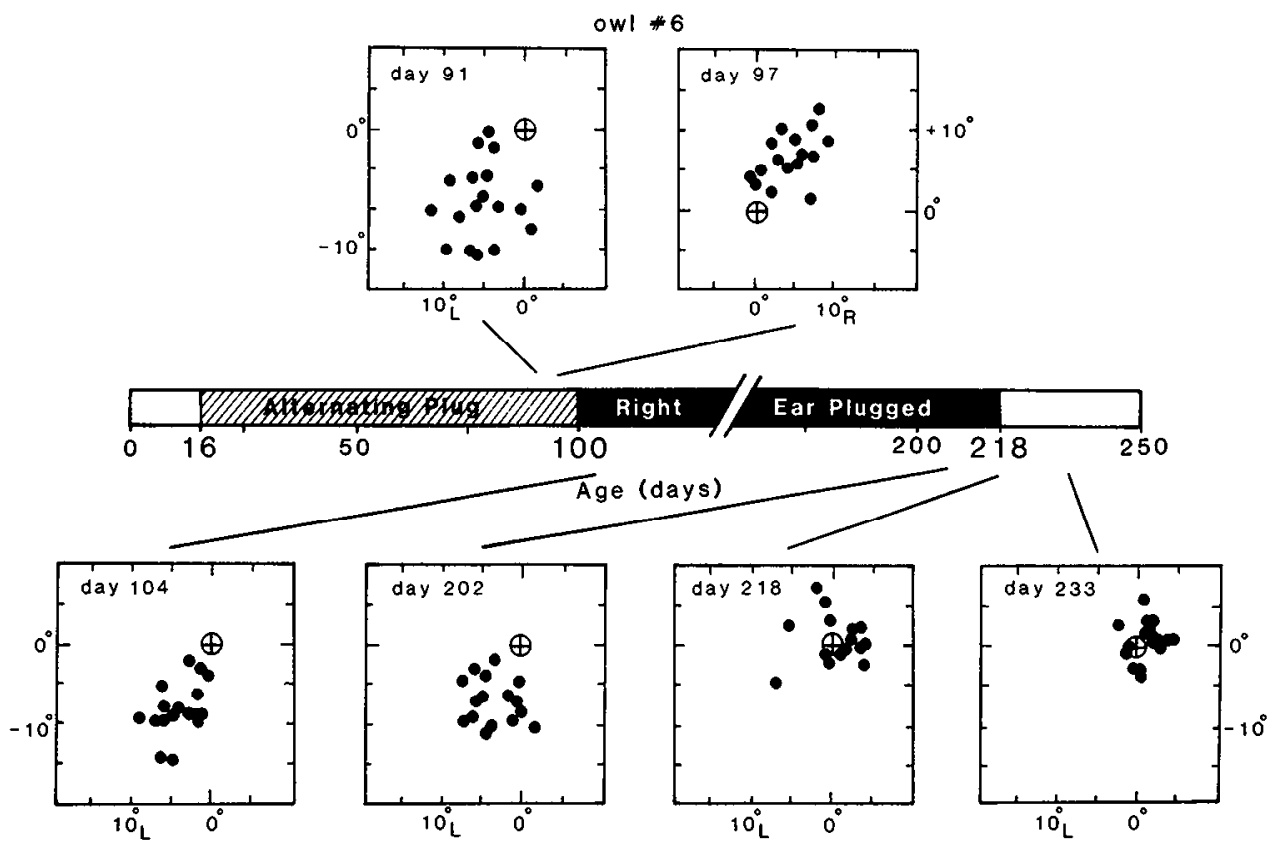

Figure 6. The effect of alternating monaural occlusion followed by chronic monaural occlusion on sound localization in owl No. 6. The boxes show the responses of the bird to auditory targets measured on particular days; the bar represents the auditory history of the animal. The top two boxes show data taken during the period of alternating monaural occlusion. On day 91, the owl's right ear was plugged; on day 97, the owl's left ear was plugged. The pattern of mean localization errors as a function of experience was similar to that shown for owl No. 3 in Figure 5. 


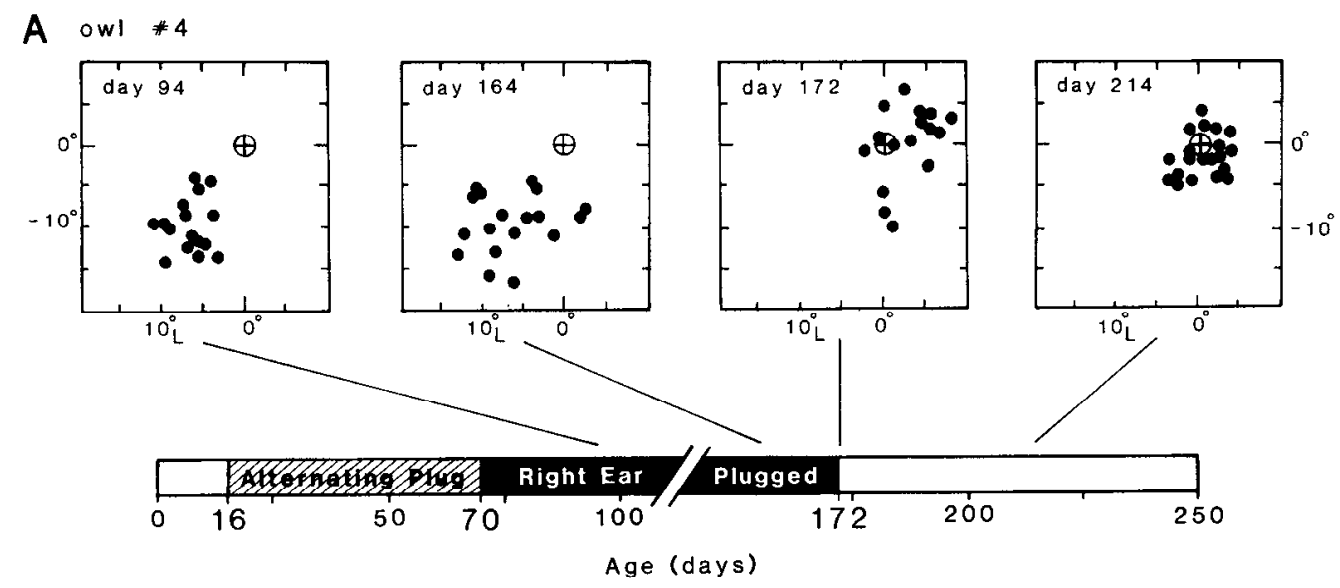

Figure 7. The effect of alternating monaural occlusion followed by chronic monaural occlusion on sound localization in owls No. 4 and 5 . The responses of owls No. $4(A)$ and $5(B)$ to auditory targets measured on particular days are shown along the top; the auditory history of each owl is represented by the bar beneath. The pattern of mean localization errors as a function of experience was similar in these birds to that shown for owl No. 3 in Figure 5. Alternating monaural occlusion followed by chronic monaural occlusion had little effect on the development of sound localization in these birds.

B owl *5

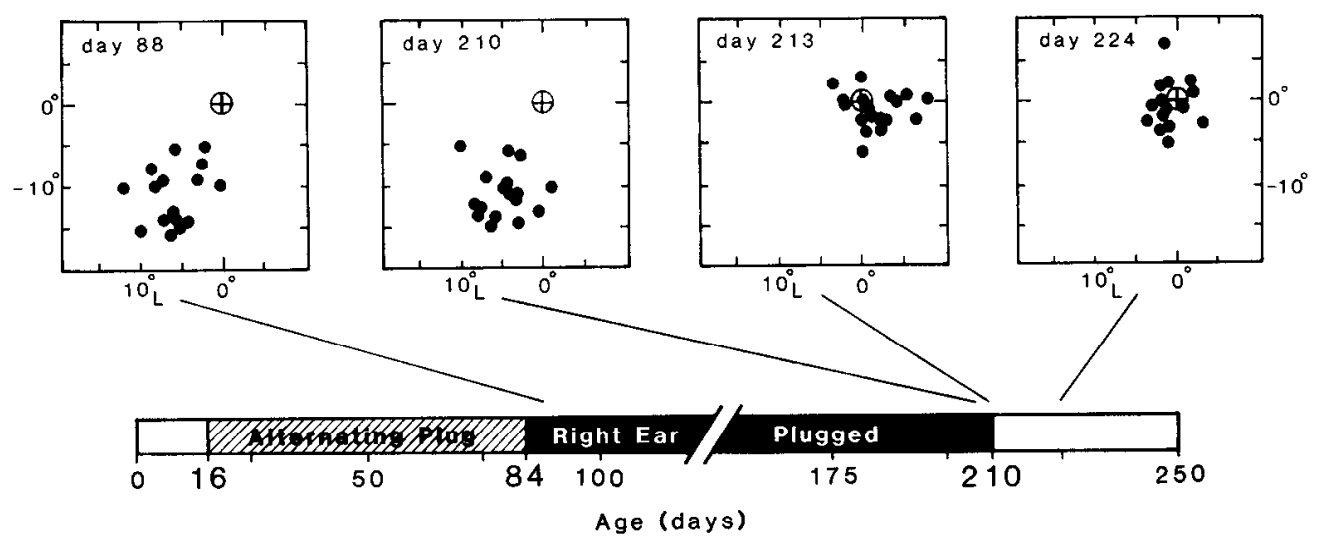

No. 2, left earplug). By $65 \mathrm{~d}$ of age (the earliest that they could be trained and tested), both owls had adjusted to their earplugs and were localizing sounds accurately (Figs. 3 and 4). When the earplug was removed from owl No. 1 at 79 d of age, it exhibited an auditory-localization error of right $9.2^{\circ}$ and up 13.4\%; when the earplug was removed from owl No. 2 at $73 \mathrm{~d}$ of age, it exhibited an auditory error of left $8.6^{\circ}$ and down $12.1^{\circ}$. (The elevational components of these errors arise because, owing to an asymmetry of the ears, the owl associates differences in interaural intensity of high frequencies with the elevation of the source; Knudsen and Konishi, 1979; Payne, 1971). Immediately after testing each bird, we sutured a new plug into the opposite ear (owl No. 1, left earplug; owl No. 2, right earplug).

After its earplug was switched, owl No. 1 was unable to localize sounds in either dimension. It responded to an auditory stimulus with multiple head saccades in various directions, the vast majority being to the right. The animal rarely fixated on one location as long as the sound continued, and the final point of fixation, when the sound ended, was not correlated with the position of the sound source.

The second (left) earplug was removed when owl No. 1 reached $127 \mathrm{~d}$ of age (Fig. 3). Surprisingly, the auditory error was right $8.1^{\circ}$ and $u p 11.5^{\circ}$, which was very close to the error measured when the first earplug had been removed $49 \mathrm{~d}$ earlier. (The direction of this error was surprising because removal of a left earplug always resulted in localization errors to the left and down in previous experiments; Knudsen, 1985; Knudsen et al., 1982, $1984 a$, b.) Following removal of the earplug, the auditory error decreased at an average rate of $0.4^{\circ}$ per day and, after $31 \mathrm{~d}$, the owl was localizing sounds with normal accuracy. The latter observation indicates that the critical period was still open in this animal.

To determine whether a longer exposure to reverse occlusion might be necessary to change sound localization in older animals, we left the right earplug of owl No. 2 in place for $178 \mathrm{~d}$, until age $250 \mathrm{~d}$ (Fig. 4). On no day during this period was the owl able to localize the auditory stimulus in either dimension: It behaved much as owl No. 1 did during reverse occlusion, cxccpt that the majority of head saccades were made to the left side. When the right earplug was finally removed at $250 \mathrm{~d}$ of age, the owl exhibited an auditory localization error of left $7.2^{\circ}$ and down $8.5^{\circ}$. (Removal of a right earplug had always resulted in errors to the right and up in previous experiments; Knudsen, 1985; Knudsen et al., 1982, 1984a, b.) This error was smaller than the one measured immediately after removal of the initial earplug, yet it was in the same direction and was remarkably large considering that the right ear had been occluded for 178 d. Thus, neither owl No. 1 nor No. 2 adjusted to a second set of abnormal auditory cues introduced after $72 \mathrm{~d}$ of age, even though these animals had never experienced normal, adult cues.

Following the removal of the right earplug from owl No. 2 at $250 \mathrm{~d}$ of age, we monitored its sound localization for 100 subsequent days (Fig. 4). In contrast to owl No. 1, owl No. 2 showed no sign of correcting its localization error; the horizontal component decreased slightly and the vertical component increased, but the total error remained constant at about $10^{\circ}$. Apparently, the abnormal auditory associations induced in owl No. 2 by the initial earplug had become permanent by $250 \mathrm{~d}$ of age despite the fact that during the preceding $178 \mathrm{~d}$ this bird was unable to localize sounds.

\section{Frequent earplug reversals}

The results described above do not establish whether or not the sensitive period ended during the initial monaural occlusion since, having adjusted to the initial monaural occlusion, the birds were apparently unable to interpret the abnormal local- 
ization cues resulting from plugging the opposite ear (see Discussion). To avoid this problem, we subjected owls No. 3-6 to monaural occlusions that were reversed every other day (Table 1). Alternating monaural occlusions began at $16 \mathrm{~d}$ of age, a time when the skull is still small, the facial ruff (the owl's external ears) has just begun to grow, and the auditory canals are about half of their adult diameters (Knudsen et al., 1984a). Alternating occlusions continued until the owls were 62-, 70-, 84-, and 100d-old, respectively, i.e., past the end of the normal sensitive period, occurring at 55-60 d of age. Alternating occlusions were followed immediately by chronic occlusion of the right ear to test for the ability of each owl to adjust to abnormal cues.

During the period of alternating monaural occlusion, all of the animals made systematic localization errors, which varied depending on the ear that was plugged. This was apparent qualitatively from the misdirected orientation responses of the baby birds to hidden and visible sound sources, and was documented quantitatively in owl No. 6, which was exposed to alternating monaural occlusion up to the age of $100 \mathrm{~d}$ (Fig. 6). Thus, these birds never adjusted completely to any set of abnormal cues during the period of alternating occlusion. Nevertheless, the birds were unable to adjust auditory-localization accuracy during the period of chronic monaural occlusion. Even owl No. 3, which was only 62-d-old when its right ear was chronically occluded, continued to exhibit a large error to the left and down for $105 \mathrm{~d}$ following chronic occlusion (Fig. 5). On the day after the chronic earplug was removed, its localization error was right $0.8^{\circ}$ and down $3.0^{\circ}$, which is not significantly different from the localization errors of normal birds. Normal localization accuracy was observed in all but one owl immediately following removal of the chronic earplug (Figs. 5, 6, and 7). Owl No. 4 exhibited a barely significant error of right $3.6^{\circ}$ and up $1.2^{\circ}$, and this error decreased to right $2.7^{\circ}$ and down $1.3^{\circ}$ in $9 \mathrm{~d}$. The localization accuracy of these birds upon removal of the chronic earplug is noteworthy because these animals had never experienced normal adult cues.

In contrast, the localization precision of each of the 4 owls was worse than normal in one or both dimensions. Upon removal of the chronic earplug, the azimuthal and elevational precisions of the owls were $3.2^{\circ}$ and $4.6^{\circ}$ for owl No. $3,3.1^{\circ}$ and $3.9^{\circ}$ for owl No. $4,3.2^{\circ}$ and $2.1^{\circ}$ for owl No. 5, and $3.4^{\circ}$ and $3.0^{\circ}$ for owl No. 6. Only the elevational precisions of owls No. 5 and 6 were within the normal ranges of $3.0^{\circ}$ for azimuth and $3.7^{\circ}$ for elevation. The precisions of all the animals improved to normal in 11 to $30 \mathrm{~d}$.

\section{Discussion}

The sensitive period is regulated by a process that is essentially age-dependent

The associations between auditory cues and locations in space that underlie sound localization are highly plastic during the first 8 weeks of life in the barn owl. We tested the hypothesis that this sensitive period is ended by sensory experience. The hypothesis was inspired by the striking coincidence between the maturation of the head and external ears at 6 weeks of age and the end of the sensitive period at 8 weeks of age (Knudsen et al., 1984a). The model proposed that the sensitive period draws to a close when the auditory system is exposed to sound localization cues that are within a particular (adult) range and the associations of these cues with locations in space are correct. The results presented here show that the sensitive period is not extended when baby owls are prevented from experiencing normal or stable auditory cues (owls No. 3-6; Figs. 5, 6, and 7). Thus, any experience-dependent model that requires exposure to accurately localized sounds for the termination of the sensitive period is no longer tenable.

However, these data cannot rule out the possibility that sensory experience plays a necessary, but secondary, role in ending the sensitive period. For example, the sensitive period might end once an owl is older than 8 weeks and its auditory cues are stable (even if abnormal) for a minimum period of time (less than $2 \mathrm{~d}$ ). According to this hypothesis, the sensitive period could be extended by total auditory deprivation (just as the visual sensitive period for monocular deprivation is extended by dark-rearing; Cynader and Mitchell, 1980; Mower et al., 1983 ) or by more rapid alternating monaural occlusions. The age-dependent prerequisite must be included because the previous study showed that the sensitive period normally ends about 2 weeks after the head and ears mature (Knudsen et al., 1984a). Without the age-dependent condition, the sensitive period would end at 6 weeks of age, immediately after the maturation of the head and ears. Therefore, we conclude that the sensitive period is regulated by a process that is essentially, if not exclusively, age-dependent.

The adjustment process results in a shift in the cue values used for sound localization

Monaural occlusion of any normal owl causes it to make systematic errors in sound localization (Knudsen and Konishi, 1979; Knudsen et al., 1982, 1984a, b). In contrast, monaural occlusion of owls No. 1 and 2, after they had adjusted to a previous occlusion of the opposite ear, rendered them unable to localize sounds at all (Figs. 3 and 4); these birds either could not evaluate or could not interpret the localization cues that resulted from the second monaural occlusion. This indicates that the auditory system is able to use only limited ranges of cue values for sound localization (for example, limited ranges of interaural time and intensity differences) and that, in adjusting to the initial monaural occlusion, the auditory system had shifted the ranges of cue values that it could evaluate or interpret. Because of this shift in ranges of cue values that it could use, the new cue values resulting from unplugging one ear and plugging the other were beyond the range the auditory system could use. It is for this reason that these data do not reveal whether or not the sensitive period had ended during the initial monaural occlusion.

\section{Innate developmental mechanisms establish approximately correct auditory associations for sound localization}

Despite being raised with abnormal auditory cues that changed drastically every other day from $16 \mathrm{~d}$ of age onward, owls No. 3-6 still developed good sound localization accuracy (Figs. 5, 6 , and 7). These birds did not experience normal adult cues until the day that the chronic earplug was removed. Yet, immediately upon removal of the earplug, each of them localized sounds accurately (although impreciscly). The associations upon which these localizations were based could not have been established or validated by experience. Hence, they must have been established by innate mechanisms that operate independently of experience.

The importance of sensory experience in the development of sound localization may be in the fine-tuning of an otherwise innate pattern of connectivity. This is suggested by the observation that, after removal of the chronic earplugs, the sound localization precision of the owls raised with alternating monaural occlusions was significantly worse than that of owls raised under normal conditions.

\section{Abnormal associations that are established by experience remain stable for long periods of time without functional validation}

Owls No. 1 and 2 altered their auditory associations to make use of the abnormal cues caused by chronic monaural occlusions imposed during the sensitive period (Figs. 3 and 4). After the earplug was switched to the opposite ear, neither bird was able to localize sounds. This condition lasted for $49 \mathrm{~d}$ in owl No. 1 and $178 \mathrm{~d}$ in owl No. 2. During this time, the abnormal associations that each bird had formed, based on the first earplug, 
remained unchanged: Upon removal of the second earplug, both birds localized sounds in the same way that they had after the first earplug was removed. This remarkable result indicates that abnormal auditory associations do not deteriorate or drift back to those associations to which the system is innately predisposed. Apparently, a mismatch between auditory and visual localization actively reshapes the associations (Knudsen and Knudsen, 1985). Our interpretation is that, during the second monaural occlusion, the birds could not interpret the auditory cues they experienced, so there was no auditory-visual spatial mismatch to generate a corrective force. The complementary experiment, preventing owls with sound localization errors from seeing, yields similar results: The owls make no adjustments of their auditory errors (Knudsen and Knudsen, 1985).

\section{The development of sound localization in barn owls}

Current and previous data support the following model of the development of sound localization in the barn owl. Soon after birth, neural connectivity supporting accurate, though imprecise, sound localization is established by innate developmental mechanisms. Within the sensitive period, this connectivity is plastic and subject to adjustment and modification by experience. The guiding force that directs this adjustment is generated by a comparison of auditory and visual spatial information. Normally, the correspondence between auditory cues and visually confirmed sound-source locations agrees with the innate associations, and sensory experience simply refines this connectivity. However, if auditory localization disagrees with visual spatial information, either because of abnormal auditory cues or altered vision, then a corrective force causes the auditory system to modify its interpretation of spatial cues until auditory localization agrees with visual localization. Bccausc the adjustment process requires many days or weeks, substantial changes in sound localization result only if a mismatch between visual and auditory space is consistent and persists for a long period of time. As the end of the sensitive period approaches, the adjustment process slows, and, by 8 weeks of age, the capability of forming abnormal associations becomes extremely limited (Knudsen et al., 1984a).

After the sensitive period ends, but within the critical period, adjustments of cue-location associations are restricted to those that are consistent with the normal correspondence of cues with locations, associations to which the auditory system is innately predisposed (Knudsen et al., 1984b). During this period of re- stricted plasticity, mismatches between auditory and visual localization continue to guide the adjustment process (Knudsen and Knudsen, 1985).

The critical period ends when neuronal connectivity becomes consolidated in its final form. Whether the critical period can be ended by normal experience during the interval between 8 and 29 weeks of age, or whether the critical period is strictly age-dependent, remains unresolved and cannot be resolved with behavioral tests: Because the sensitive period has ended, new abnormal associations cannot be formed, whether the normal associations have been consolidated or not; the only adjustments possible are from abnormal toward normal associations. Although, to the animal, the inability to form abnormal associations and the consolidation of normal associations are functionally equivalent, mechanistically they may be quite distinct events. To resolve this problem, one must identify the neuronal events that end the critical period and determine whether normal experience can precipitate their occurrence before they are triggered unconditionally at about 29 weeks of age.

\section{References}

Cynader, M., and D. E. Mitchell (1980) Prolonged sensitivity to monocular deprivation in dark-reared cats. J. Neurophysiol. 4: 10261040.

Knudsen, E. I. (1985) Experience alters the spatial tuning of auditory units in the optic tectum during a sensitive period in the barn owl. J. Neurosci. 5: 3094-3109.

Knudsen, E. I., and P. F. Knudsen (1985) Vision guides the adjustment of auditory localization in young barn owls. Science $230: 545-548$.

Knudsen, E. I., and M. Konishi (1979) Mechanisms of sound localization by the barn owl (Tyto alba). J. Comp. Physiol. 133: 13-21.

Knudsen, E. I., P. F. Knudsen, and S. D. Esterly (1982) Early auditory experience modifies sound localization in barn owls. Nature 295: 238240.

Knudsen, E. I., S. D. Esterly, and P. F. Knudsen (1984a) Monaural occlusion alters sound localization during a sensitive period in the barn owl. J. Neurosci. 4: 1001-1011.

Knudsen, E. I., P. F. Knudsen, and S. D. Esterly (1984b) A critical period for the recovery of sound localization accuracy following monaural occlusion in the barn owl. J. Neurosci. 4: 1012-1020.

Mower, G. D., W. G. Christen, and C. J. Caplan (1983) Very brief visual experience eliminates plasticity in the cat visual cortex. Science 221: $178-180$.

Payne, R. S. (1971) Acoustic location of prey by barn owls (Tyto alba). J. Exp. Biol. 54: 535-573. 